\title{
FPGA based laser frequency stabilization using FM spectroscopy
}

Christian Spindeldreier, Wolfgang Bartosch, Thijs Wendrich, Ernst M. Rasel, Wolfgang Ertmer, et al.

Christian Spindeldreier, Wolfgang Bartosch, Thijs Wendrich, Ernst M. Rasel, Wolfgang Ertmer, Holger Blume, "FPGA based laser frequency stabilization using FM spectroscopy," Proc. SPIE 10518, Laser Resonators,

Microresonators, and Beam Control XX, 1051813 (16 February 2018); doi: $10.1117 / 12.2288370$

SPIE. Event: SPIE LASE, 2018, San Francisco, California, United States 


\title{
FPGA based Laser Frequency Stabilization using FM-Spectroscopy
}

\author{
Christian Spindeldreier ${ }^{a}$, Wolfgang Bartosch ${ }^{b},{\text { Thijs } \text { Wendrich }^{b} \text {, Ernst M. Rasel }}^{b}$, Wolfgang \\ Ertmer $^{b}$, Holger Blume ${ }^{a}$ \\ ${ }^{a}$ Leibniz Universität Hannover, Faculty of Electrical Engineering and Computer Science, \\ Institute of Microelectronic Systems, Appelstr. 4, 30167 Hannover, Germany \\ ${ }^{b}$ Leibniz Universität Hannover, Faculty of Mathematics and Physics, Institut für \\ Quantenoptik, Welfengarten 1, 30167 Hannover, Germany
}

\begin{abstract}
Frequency stabilized light sources with narrow linewidth are mandatory for atom interferometry based experiments. For compact experiment designs used on space platforms, tunable DFB diode lasers are often used. These lasers combine low energy consumption with small sizes, but lack long-term frequency stability. This paper presents an FPGA based laser frequency stabilization system for highly variable target frequencies using frequency modulated Rb-spectroscopy achieving latencies below $100 \mu \mathrm{s}$. The system consists of a DFB laser, a Rb-spectroscopy cell, a laser current controller and an FPGA board with an analog-digital conversion board. The digital part of the frequency stabilization system is a SoC mapped on an FPGA. The SoC consists of a processor, enabling user interaction via network connection, and the dedicated frequency stabilization module. This module consists of a demodulation stage, digital filters, a frequency estimator and a controller. To estimate the frequency, small ramps of the laser frequency are generated using a high-speed DAC connected to the laser current controller. The absorption spectroscopy output of this beam is sampled using a photodiode and a high-speed ADC. After signal conditioning with digital filters, the frequency estimator extracts the present mid-frequency of the laser applying pattern matching with a prerecorded reference spectrum. The frequency controller adjusts the mean laser current based on this estimation. The performance as well as the accuracy of the proposed laser stabilization system and its FPGA resource and power consumption are evaluated.
\end{abstract}

Keywords: FPGA, System on Chip, distributed feedback laser, frequency control, frequency stabilization, optical spectrum, signal recognition, absorption spectroscopy

\section{INTRODUCTION}

Atom interferometers are powerful tools to test fundamental physical principles and measure physical quantities like acceleration, rotation, gravity gradients as well as time with a very high precision. ${ }^{1}$ A two species atom interferometer allows a high precision test on the equivalence principle, one of the key assumptions of Einsteins theory of general relativity, with test masses that obey the laws of quantum mechanics. ${ }^{2}$ The result of this type of experiment can give a hint, how a unified theory of quantum mechanics and general relativity theory might look like. ${ }^{3}$ To test the equivalence principle, the local gravity is calculated from a phase-measurement for two different species with an atom interferometer. The measured relative phase $\Delta \Phi$ in such an experiment is dependent on the square of the free evolution time $T$ in the interferometer:

$$
\Delta \Phi=\vec{k}_{e f f} \cdot \vec{a} \cdot T^{2}
$$

where $\vec{k}_{\text {eff }}$ is the photons wave vector and $\vec{a}$ the local acceleration. ${ }^{4}$ Since a free falling ensemble of atoms on ground can only fall until the bottom of the experimental chamber, the accurancy of these experiments can be improved by moving them to microgravity environments. These kind of experiments are performed by the

Address all correspondence to: Christian Spindeldreier, E-mail: spindeldreier@ims.uni-hannover.de, Tel: +49 (0)511-762-5039, Fax: +49 (0)511-762-19601

\footnotetext{
Laser Resonators, Microresonators, and Beam Control XX, edited by Alexis V. Kudryashov,

Alan H. Paxton, Vladimir S. Ilchenko, Proc. of SPIE Vol. 10518, 1051813 · @ 2018 SPIE

CCC code: $0277-786 X / 18 / \$ 18 \cdot$ doi: $10.1117 / 12.2288370$
} 
QUANTUS collaboration at the drop tower in Bremen, Germany, reaching microgravity levels below $10^{-4} \mathrm{~g}$ for up to 9 seconds. $^{5}$ Further extension of the precision of the atom interferometry experiments require extended microgravity times which can only be achieved on space platforms. In january 2017 the QUANTUS team carried out an interferometry experiment on board of a VSB-30 sounding rocket within the MAIUS-1 mission. The following MAIUS sounding rocket missions as well as the BECCAL experiment, a cooperation between QUANTUS collaboration and NASA JPL on board of the international space station will allow equivalence principle tests based on quantum test masses at new levels of precision.

Atom interferometry requires light of specific wavelengths to manipulate atomic states. This light has to be precisely controlled to a fraction of an atomic transitions natural linewidth to enable precise manipulation of the atomic state. ${ }^{6}$ These linewidths are in the order of several $\mathrm{MHz}$ for alkali atoms like potassium and rubidium, commonly used for atom interferometry.

Space platforms are always limited in the available volume and power, therefore tunable laser diodes are used as light sources. The light, these type of diodes provide, remains sufficiently stable for short periods of time $(<1 \mu \mathrm{s})$, but lack of long term stability. Due to the sensitiveness to injection current, temperature, acoustics and mechanical strain frequency shifts of several linewidths can be caused at timescales beyond $1 \mathrm{~ms}^{7}$ To suppress these noise effects wavelength stabilization to an absolute reference is mandatory.

A variety of stabilization methods exists, like optical cavities or beat measurements, but only the use of atomic transitions in absorption spectroscopy is sufficient to enable absolute frequency referencing. ${ }^{8}$ In a spectroscopy the laser wavelength is scanned over a certain frequency range and sent through a gas cell. Atomic transitions cause spectral features with characteristic absorption and dispersion signals at specific wavelengths being natural constants. By modulating the phase of the laser beam in the range of several $\mathrm{MHz}$, the sensitivity of the spectroscopy is enhanced. This spectroscopy variant is called frequency modulated (FM) spectroscopy. The distortion of the probe wave through atomic transitions is converted to a zero crossing in the FM spectroscopy output signal as shown in Figure $1 .{ }^{9}$

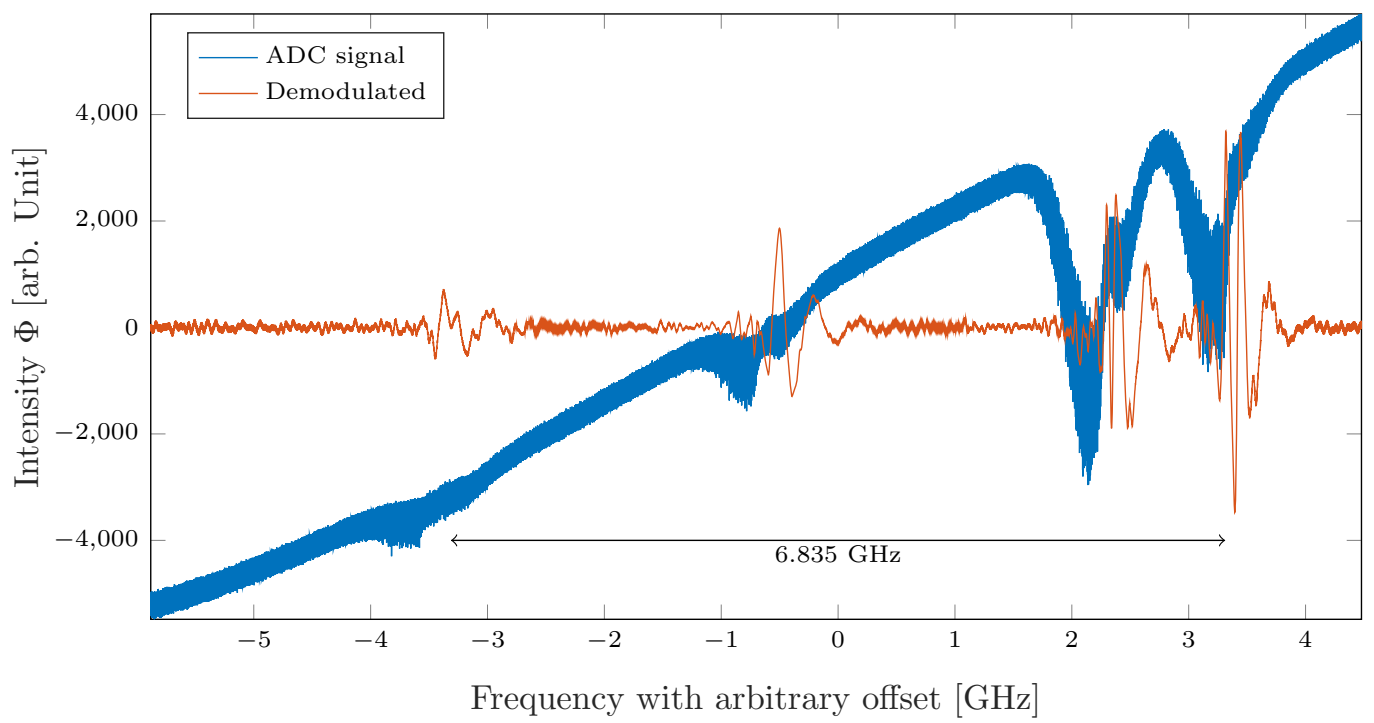

Figure 1: Raw FM spectroscopy signal (blue) and demodulated spectroscopy signal (red) of ${ }^{85} \mathrm{Rb}$ and ${ }^{87} \mathrm{Rb}$ D2-Line

This signal shape enables the use of a PID controller to stabilize the lasers wavelength to the spectral feature, but the error signal is limited to the natural linewidth of the atomic transition. Therefore, a PID controller using the FM spectroscopy signal as error signal requires manual preselection of the atomic transition and is vulnerable to frequency shifts bigger than the transitions linewidth.

Field programmable gate arrays (FPGA) are more and more used within laser locking devices. While one device uses an FPGA to enable phase locking of two lasers, ${ }^{10}$ others are used to lock external cavity diode lasers 
(ECDL) to external cavities. ${ }^{11,12}$ Recently, an FPGA based system has been presented, which uses frequency modulated spectroscopy to lock a ECDL to an absolute reference. ${ }^{13}$ This system is based on the above mentioned PID controlling principle and requires manual preselection of the desired atomic transition which is not suitable for experiments on space platforms.

Therefore, a custom laser wavelength estimation algorithm was developed for the MAIUS sounding rocket experiment. The algorithm combines high precision FM absorption spectroscopy with signal matching algorithms. Transferring the algorithm to an FPGA reduces communication latency and paves the way to use the estimation algorithm for real time wavelength stabilization and controlling. ${ }^{14}$ This paper presents a complete wavelength stabilization system based on a wavelength estimation module. The signal processing chain consists of spectroscopy input signal generation, demodulation, wavelength estimation and controlling.

The remainder of this paper is structured as follows: Section 2 presents the physical setup of the wavelength stabilization system including a demonstration laser. The wavelength estimation and stabilization principle is described in Section 3. The digital signal processing system mapped on the FPGA is explained in Section 4 whereas Section 5 presents the evaluation of the system comprising frequency controlling accuracy as well as FPGA resource and energy consumption. Conclusions are drawn in Section 6.

\section{PHYSICAL SYSTEM}

The physical part of the proposed FPGA based laser frequency stabilization system is shown in Figure 2 . It consists of a DFB laser module with center wavelength of $780 \mathrm{~nm}$ custom built for the QUANTUS project. The laser is connected to an optical isolator suppressing any back-reflection in the laser diode. The laser beam is then separated into a spectroscopy beam and a work beam which is used within the experimental setup, for example as reference beam for offset locked high power lasers.

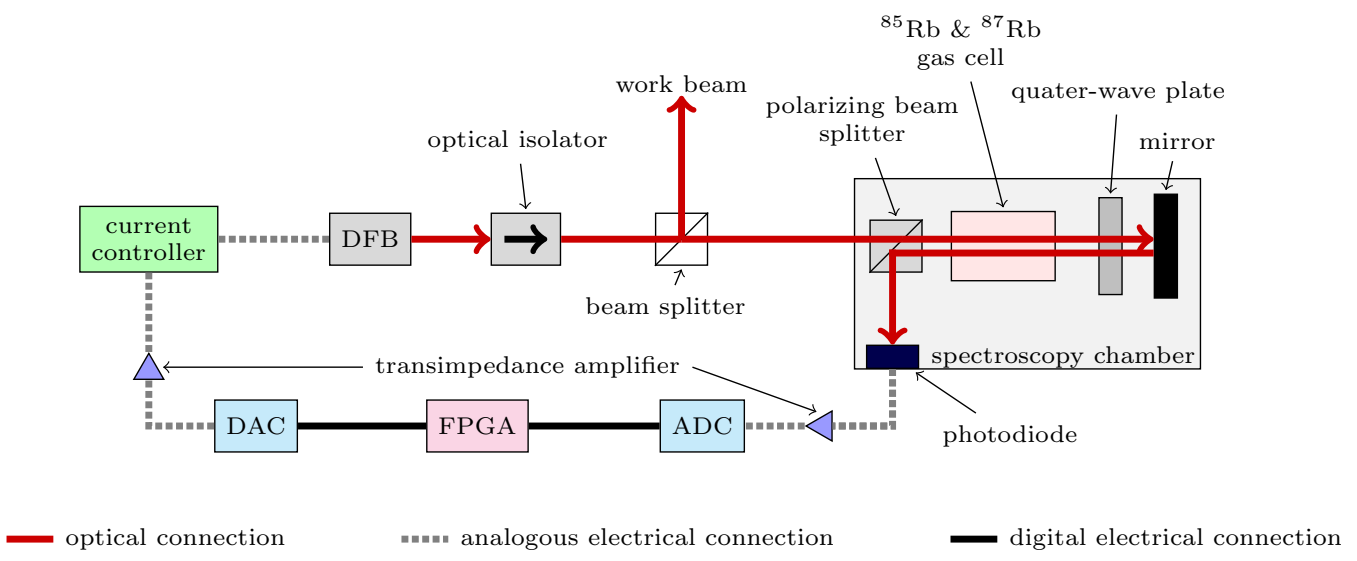

Figure 2: Physical setup of wavelength stabilisation system

The spectroscopy beam is guided to the spectroscopy chamber. There it passes a polarizing beam splitter which enables adjusting the power of the laser beam which is transmitted through a gas cell containing ${ }^{85} \mathrm{Rb}$ and ${ }^{87} \mathrm{Rb}$ isotopes. As shown in Figure 2 the beam then passes through a quarter-wave plate and is reflected by a mirror back through the plate. By passing the waveplate twice the polarization axis of the reflected beam is shifted. After passing the gas cell again, the beam is reflected by the beam splitter due to its shifted polarization axis. This reflected beam is guided to a PIN-photodiode which converts the optical signal to a current signal. As the temperature of a tunable laser diode affects the wavelength the diode temperature is held constant with Peltier elements driven by an external temperature controller.

The current signal of the photodiode is converted to a voltage signal by a transimpedance amplifier which is connected to a high speed ADC with a sampling rate of $50 \mathrm{MS} / \mathrm{s}$ and a resolution of 14 bit. The ADC is capacitatively coupled to the transimpedance amplifier to remove the DC offset of the spectroscopy signals. This enables the usage of the ADC's full resolution for spectroscopy features. 
The ADC and its amplifier are located on a custom extension card for the Altera DE2-115 board. The digital interface of the ADC is connected to the Intel Cyclone IV FPGA on the DE2-115 board. The signal processing system containing laser frequency estimation and controlling modules as described in Section 4 is mapped on this FPGA. Next to the ADC there is a high speed DAC on the extension card connected to the FPGA too. It uses the same sample rate and resolution as the ADC. The DAC is connected to another transimpedance amplifier which generates the DFB laser diodes input signal, closing the feedback loop. As a laser diode requires a certain DC offset current there is an external current controller which provides an offset current between $30 \mathrm{~mA}$ and $140 \mathrm{~mA}$ depending on the connected laser diode. It contains a modulation input where the DAC signal is added to the offset current. The sum signal is directly connected to the DFB laser diode.

\section{WAVELENGTH ESTIMATION AND STABILIZATION PRINCIPLE}

An accurate estimation of the wavelength is mandatory to lock a laser diode to a user defined frequency. Therefore, frequency modulated spectroscopy is chosen as the basic principle. The diode current is modulated with a slow triangle wave with a frequency of $48.8 \mathrm{kHz}$ and an amplitude of $160 \mu \mathrm{A}$. This slow ramp produces a continuous wavelength change in the light emitted by the DFB laser diode. As the wavelength is dependent on the DFB laser current, the change of the wavelength follows the triangle wave. Additionally, a sinusoidal wave at higher frequency $(6.25 \mathrm{MHz})$ and higher amplitude $(430 \mu \mathrm{A})$, is added to the triangle wave of the laser input current, generating the diode current shown in Figure 3a.

As the modulation index $M$ is low $(M \ll 1)$, the electric field component of the light emitted by the laser diode can be approximately described by:

$$
E_{\text {laser }}(t) \approx E_{0}\left(\frac{M}{2} e^{j\left(\omega_{c}-\omega_{m}\right) t}+e^{j \omega_{c} t}+\frac{M}{2} e^{j\left(\omega_{c}+\omega_{m}\right) t}\right)
$$

where $E_{0}$ is the electric field amplitude of the laser signal. Two weak sidebands at $\omega_{c} \pm \omega_{m}$ around the carrier signal are generated. ${ }^{9}$ Due to the slow triangle wave the frequency of the carrier signal $\omega_{c}$ changes slowly following the current triangle. When the modulated beam interacts with spectral features individual attenuation caused by intensity absorption and phase shift induced by dispersion of both sidebands can be observed.

The photodiode current is proportional to the intensity envelope impinging on the diode. The current can be approximately described with:

$$
I_{\text {photo }}(t) \approx I_{0}\left(1-\Delta \delta M \cos \left(\omega_{m} t\right)+\Delta \phi M \sin \left(\omega_{m} t\right)\right)
$$

where $\Delta \delta$ describes the deviation from the background loss induced by absorption trough the spectral feature whereas $\Delta \phi$ describes the deviation induced by the dispersion caused by the spectral features. ${ }^{9}$ Equation 3 shows that the diode current contains signal components which are proportional to the absorption and dispersion through the spectral feature. Extracting these signal components from the photodiode signal enables the detection of the hyper-fine structure of rubidium isotopes. ${ }^{15}$

The scan current called modulated current, shown in Figure 3a, results in a scan signal obtained from the photodiode, shown in Figure 3b. As the information of the rising ramp and the falling ramp are the same, the signal from the photodiode is recorded only on the rising ramp. After recording the signal has to be demodulated. Demodulation is realized by mixing the recorded signal with the sinusoidal modulation frequency. A bandpass filter is applied to the mixed signal resulting in the signal shown in Figure 3b. The signal contains visible features as a result of the absorption and dispersion of the laser light by atomic transitions.

Next to these scan signals there is a complete spectrum signal, shown in Figure 1, containing all features of ${ }^{85} \mathrm{Rb}$ and ${ }^{87} \mathrm{Rb}$. It is recorded by setting the maximum and minimum values of the triangle wave to the limits of the DAC while keeping the slope from the scan signal as described above. As the features of the spectrum are directly related to the atomic transition frequencies of rubidium isotopes being natural constants, the spectrum is suitable as an absolute reference signal for wavelength stabilization.

To estimate the wavelength, the position of the scan signal has to be found in the reference spectrum signal. A pattern matching algorithm using the mean scan current to preselect the part of the spectrum is used to find 


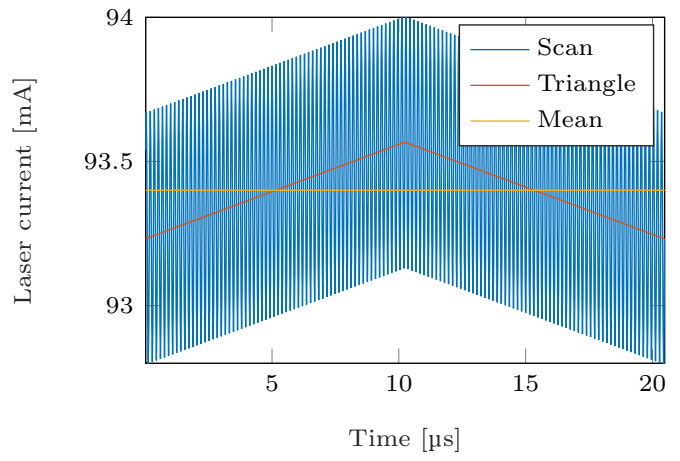

(a) Laser current signal

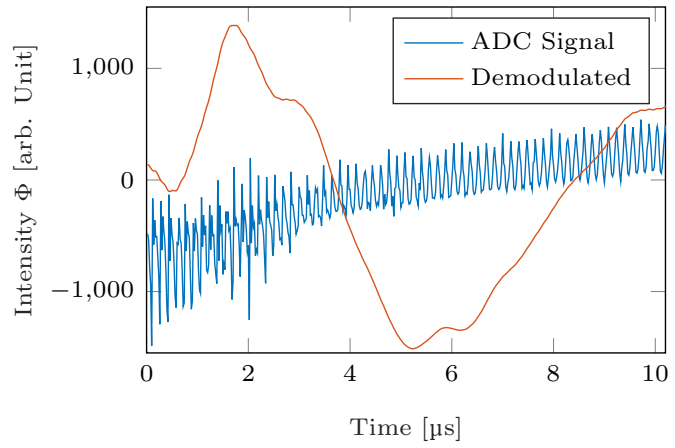

(b) Photodiode signal

Figure 3: Laser current scan signal (a) and the signal measured by the photodiode (b)

the position of the scan. ${ }^{14}$ The estimated position represents the mean wavelength of the processed scan signal. This information is used within a PI controller which controls the mean value of the scan current, closing the wavelength control loop. The target wavelength is given as its corresponding position in the reference spectrum. Due to the above mentioned limited long term stability of diode lasers, the described control loop has to be executed within not more than $100 \mathrm{\mu s}$. As the scan must contain at least one spectral feature, but the position of the feature within the scan is variable, the target frequency is variable around a feature within the scan frequency range of about $300 \mathrm{MHz}$.

\section{DIGITAL SYSTEM}

To fulfill the stringent real-time constraints of the above described wavelength estimation principle and controlling loop, a dedicated hardware module is used. This module is integrated in a System on a Chip (SoC), mapped on an FPGA, comprising a LEON3 sparc processor, a memory controller (MCTRL) and an ethernet controller (GRETH). All components except the frequency stabilization module are obtained from the open-source GRLIB IP Library. ${ }^{16}$ The parts of the System on Chip including the details of the frequency stabilization module are shown in Figure 4.

\subsection{Laser Frequency Stabilization Hardware Module}

The ADC signal containing the converted photodiode current is connected to a mixer extracting the modulation carrier from the input signal as described in Section 3. To remove inter-modulation and noise signal components a biquad filter with a configurable order is used. The resulting signal is stored in the scan RAM block comprising 512 samples. Scan signal recording is synchronized to the lower turning point of the triangle wave shown in Figure 3a to store only the rising part of the triangle. As shown in Figure 4 there are two multiplexers in the input signal path which can be used to record the raw or filtered scan signal for offline processing and evaluation. Furthermore, the signal can be stored in the spectrum RAM comprising 65536 samples enabling reference spectrum recording using the same setup. During normal operation mode, the spectrum RAM contains a prerecorded spectrum which is separated in four regions of interest containing the spectral features to reduce the computational complexity for the wavelength estimator. The region, the present scan is searched in, is selected by its mean scan current. After storing the scan signal to its RAM block, the wavelength estimator determines the position of the present scan in the reference spectrum using a scalable hardware module which realizes the sum-of-absolute-differences (SAD) pattern matching algorithm. The internal architecture including all details is described by Spindeldreier et al. ${ }^{14}$

The estimated position is used as the process value for the PI-controller while the position derived from the user defined target wavelength is the setpoint. The control value is the mean current of the triangle wave. The adjusted mean scan current is transferred to the scan controller which generates the modulated scan wave shown in Figure 3a. To avoid jumps in the laser current the scan controller adopts a new mean current value when the old mean value is passed. With the new mean value updated upper and lower turning points are determined and the scan ramp will be extended to this new turning points. 


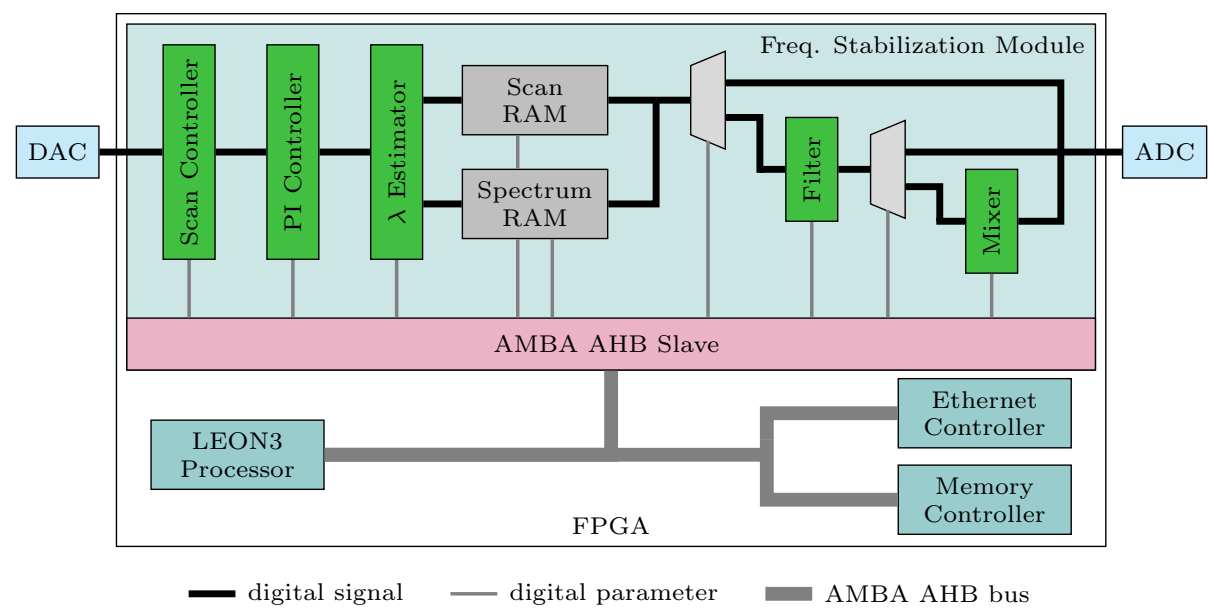

Figure 4: System on a Chip for laser frequency stabilization

\subsection{System on a Chip}

The above explained laser frequency stabilization module contains an Advanced High-performance Bus (AHB) slave interface. ${ }^{16}$ The interface is connected to the AHB network of the System on a Chip, shown in Figure 4, enabling the adjustment of several parameters like the wavelength setpoint, region current separators, PI gains or filter coefficients. Furthermore the scan and spectrum data can be transferred from or to the module. In order to interact with the $\mathrm{SoC}$, it is connected to a Host-PC via ethernet. The LEON3 Processor acts as the master in the SoC initiating data transfer and handling the network data. Therefore, the embedded real time operating system RTEMS $4.11^{17}$ runs on the LEON3. A custom RTEMS driver allows access to all parameters of the frequency stabilization module. Next to a serial terminal interface, a custom TCP based network protocol is employed for parameter and data transfer. A Qt based GUI is used on a Host-PC for parameter changing as well as data visualization and export.

\section{EVALUATION}

To evaluate the performance of the presented laser frequency stabilization system the mean scan current as well as the estimated scan frequency are analyzed. Furthermore, the internal timing of the frequency controlling module is presented. The FPGA resource as well as the energy consumption of the whole system are examined.

\subsection{Frequency Precision}

To evaluate the frequency precision of the system without external reference, the internal estimated position which can be read as the actual wavelength next to the corresponding mean scan current is recorded during frequency controlling. The data shown in Figure 5 were recorded while the target position was set to the relative frequency of $-586.6 \mathrm{MHz}$, which is the spectral feature in the center of Figure 1.

The demodulated FM spectroscopy signal observed at this position is shown in Figure 3b. The position can be controlled very accurately, the mean estimated frequency is $-585.8 \mathrm{MHz}$ and the maximum frequency difference is $12.0 \mathrm{MHz}$. As Figure 5 shows, the mean current is changed due to the small variations in the estimated frequency. The controller keeps the frequency at a constant level while the mean laser current required to generate this frequency slowly drifts.

During evaluation of the system, the definition of the filter parameter turned out to be the most challenging task. A low-pass component is required to remove the Doppler-broadened absorption effects, the high-pass component removes inter-modulation signal components and reduces the system noise. A sixth order biquad band-pass filter with a pass-band reaching from $100 \mathrm{kHz}$ to $600 \mathrm{kHz}$ was chosen. The PI controller gains were tuned manually, however, the integral gain significantly dominates the controller behavior. 


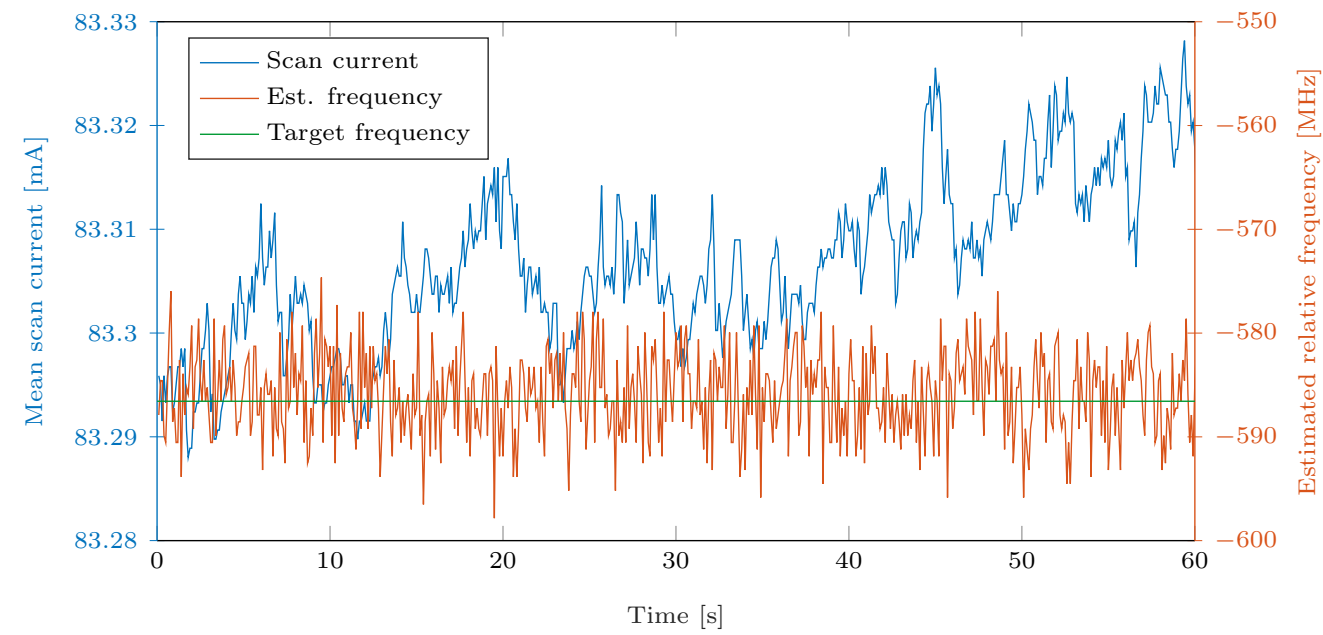

Figure 5: Mean scan current and estimated laser frequency during frequency controlling

\subsection{Timing of the Control Loop}

As the number of parallel SAD cores inside the wavelength estimation module is variable, but influences the system performance, ${ }^{14}$ the number was set to 300 cores which is slightly more than half the maximum number for all further evaluations. The whole system is clocked with $50 \mathrm{MHz}$, while the critical path is within the LEON3 processor. This configuration results in an overall controlling loop period of $82.08 \mathrm{\mu s}$.

Table 1: Partition and execution times of the frequency stabilization control loop

\begin{tabular}{l|r|l} 
Control State & Duration & Description \\
\hline Record & $10.26 \mu \mathrm{s}$ & Record scan signal \\
Estimate & $57.20 \mu \mathrm{s}$ & Estimate the position of scan signal \\
PI Control & $0.10 \mu \mathrm{s}$ & Adjust mean scan current \\
Wait & $14.52 \mu \mathrm{s}$ & Wait for start of rising scan ramp
\end{tabular}

As shown in Table 1 the control loop is split into 4 steps: During the first step the scan signal containing 512 samples is recorded. Due to the sampling frequency of $50 \mathrm{MHz}$ this operation takes $10.26 \mu \mathrm{s}$. Next the estimator module determines the position of the recorded scan within the spectrum. Due to the number of 2000 samples within a spectrum region in the actual configuration, this step lasts for $57.2 \mu \mathrm{s}$. Next the PI algorithm is executed in $0.1 \mu \mathrm{s}$ and finally the mean scan current is adjusted and the system waits for the next rising triangle ramp requiring additional $14.52 \mu \mathrm{s}$. Changing the system configuration in terms of the number of samples within a scan or the number of samples in a spectrum region affects the system performance. However, in the presented configuration the system meets the performance requirement of a control loop time below $100 \mu \mathrm{s}$.

\subsection{FPGA Resources}

The frequency stabilization System on a Chip is mapped on an Altera DE2-115 evaluation board equipped with an Intel Cyclone IV (EP4CE115F29C7) FPGA. The results presented below are obtained from the reports generated during mapping with Quartus Prime 17.1 Standard Edition from Intel FPGA.

Table 2: SoC total resource consumption and percentage of available resources of an Intel EP4CE115 FPGA

\begin{tabular}{c|c|c|c|c} 
LEs & ALUTs & Registers & DSP-Blocks (9x9) & Memory Blocks (M9K) \\
\hline $73,595(64 \%)$ & $60,671(54 \%)$ & $39,929(35 \%)$ & $182(35 \%)$ & $226(52 \%)$
\end{tabular}

Table 2 shows the total number of required FPGA resources next to the percentage of the total available resources of the used FPGA. The complete system requires about $65 \%$ of the available Logic Elements (LEs) on this FPGA. 
Table 3 provides a closer look at the distribution of the required FPGA resources to the main modules of the System on Chip. There are two main modules, laser frequency stabilization module and the LEON3 processor consuming most resources.

Table 3: Resource consumption of the internal modules and percentage of total SoC

\begin{tabular}{|c|c|c|c|c|c|c|c|c|}
\hline Module & \multicolumn{2}{|c|}{ ALUTs } & \multicolumn{2}{|c|}{ Registers } & \multicolumn{2}{|c|}{ DSP-Blocks $(9 \times 9)$} & Memory & Blocks \\
\hline Leon3 & 11,579 & $(19 \%)$ & 5478 & $(14 \%)$ & 8 & $(4 \%)$ & 80 & $(4 \%)$ \\
\hline GRETH & 2,804 & $(5 \%)$ & 116 & $(14 \%)$ & 0 & $(0 \%)$ & 6 & $(2 \%)$ \\
\hline MCTRL & 784 & $(1 \%)$ & 444 & $(1 \%)$ & 0 & $(0 \%)$ & 0 & $(0 \%)$ \\
\hline $\begin{array}{l}\text { Frequency } \\
\text { Stabilization }\end{array}$ & 41,792 & $(69 \%)$ & 31632 & $(79 \%)$ & 174 & $(96 \%)$ & 140 & $(62 \%)$ \\
\hline
\end{tabular}

The frequency stabilization module occupies about $70 \%$ of the SoC, within this module the wavelength estimator dominates the resource consumption. Overall there is space for further extensions of the system. As the system is now based on an evaluation board a smaller device can be chosen for the final laser frequency stabilization device.

\subsection{Power Consumption}

The input current and voltage of the whole frequency stabilization system were individually measured for all system components. In total the complete system consumes $23.7 \mathrm{~W}$ power during operation. The FPGA evaluation board with the attached custom extension board containing the high speed ADC and DAC requires with $9.4 \mathrm{~W}$ which is about $40 \%$ of the total power budget. The analogous part of the system comprising the laser current driver, providing an offset current of $76.2 \mathrm{~mA}$ for the DFB laser, the temperature controller and the transimpedance amplifier for the photodiode consume a total power of $14.3 \mathrm{~W}$. The power consumption shows, that the power consumption of the laser and its electronics dominates the power budget. Due to various unused components on the DE2-115 evaluation board a final system will consume less power as the simulation using Quartus Power Analyser estimates an FPGA thermal power dissipation of $900 \mathrm{~mW}$. Therefore, the presented digital laser frequency stabilization system is suitable to be used on platforms with a reduced power budget.

\section{CONCLUSION}

In this paper a FPGA based digital laser frequency stabilization system is presented. The system uses the signal form generated by laser FM spectroscopy in combination with a pattern matching algorithm to estimate the actual laser frequency. Based on this, the frequency is stabilized to a variable, user defined target frequency by a digital PI controller. The whole signal processing is performed on an Intel Cyclone IV FPGA resulting in a control loop time of $82 \mu \mathrm{s}$ requiring $65 \%$ of the available FPGA resources. A complete test system comprising a DFB laser as well as a Rb spectroscopy chamber is used to test the system accuracy and performance. The controlling accuracy is evaluated using the internal wavelength estimation as well as the mean laser diode current showing a good performance. Further evaluation using an external stable reference laser have to be performed to confirm the systems performance and accuracy. Additionally, the controlling performance might be improved by replacing the PI controller by model predicted control principles.

\section{ACKNOWLEDGMENTS}

The authors would like to thank V. Kuswik and A. Friesen for their contributions, as well as the QUANTUS team for their support. This work is supported by the German Space Agency (DLR) with funds provided by the German Federal Ministry of Economic Affairs and Energy (BMWi) under grant number 50WP1431.

\section{REFERENCES}

[1] Cronin, A. D., Schmiedmayer, J., and Pritchard, D. E., "Optics and interferometry with atoms and molecules," Rev. Mod. Phys. 81, 1051-1129 (Jul 2009).

[2] Rudolph, J., Gaaloul, N., Singh, Y., et al., "Degenerate quantum gases in microgravity," Microgravity Science and Technology 23, 287-292 (Jun 2011). 
[3] Aguilera, D. N., Ahlers, H., Battelier, B., et al., "STE-QUEST-test of the universality of free fall using cold atom interferometry," Classical and Quantum Gravity 31(11), 115010 (2014).

[4] Bordé, C. J., "Quantum theory of atom-wave beam splitters and application to multidimensiona atomic gravito-inertial sensors," General Relativity and Gravitation 36, 475-502 (Mar 2004).

[5] Müntiga, H., Ahlers, H., Krutzik, M., et al., "Interferometry with bose-einstein condensates in microgravity," Physical Review Letters 110, 093602 (Feb 2013).

[6] Metcalf, H. J. and van der Straten, P., [Laser Cooling and Trapping], Graduate Texts in Contemporary Physics, Springer New York (2001).

[7] Paschotta, R., Telle, H. R., and Keller, U., [Noise of Solid-State Lasers], vol. 119 of Optical science and engineering, 473-510, Taylor \& Francis (2007).

[8] Gawlik, W. and Zachorowski, J., "Stabilization of diode-laser frequency to atomic transitions," Optica Applicata 34(4), 607-618 (2004).

[9] Bjorklund, G. C., Levenson, M. D., Lenth, W., and Ortiz, C., "Frequency modulation (FM) spectroscopy," Applied Physics B 32(3), 145-152 (1983).

[10] Xu, Z., Huang, K., and Lu, X., "A digital optical phase-locked loop based on field programmable gate array and its applications," in [2014 International Conference on Information Science, Electronics and Electrical Engineering], 2, 795-799 (April 2014).

[11] Schwettmann, A., Sedlacek, J., and Shaffer, J. P., "Field-programmable gate array based locking circuit for external cavity diode laser frequency stabilization," Review of Scientific Instruments 82(10), 103103 (2011).

[12] Yang, G., Barry, J. F., Shuman, E. S., Steinecker, M. H., and DeMille, D., "A low-cost, fpga-based servo controller with lock-in amplifier," Journal of Instrumentation 7(10), P10026 (2012).

[13] Jørgensen, N. B., Birkmose, D., Trelborg, K., Wacker, L., Winter, N., Hilliard, A. J., Bason, M. G., and Arlt, J. J., "A simple laser locking system based on a field-programmable gate array," Review of Scientific Instruments 87(7), 073-106 (2016).

[14] Spindeldreier, C., Wendrich, T., Rasel, E. M., Ertmer, W., and Blume, H., "Fpga-based frequency estimation of a dfb laser using rb spectroscopy for space missions," in [2016 IEEE 27th International Conference on Application-specific Systems, Architectures and Processors (ASAP)], 207-212 (Jul 2016).

[15] Nakanishi, S., Ariki, H., Itoh, H., and Kondo, K., "Frequency-modulation spectroscopy of rubidium atoms with an algaas diode laser," Optics Letters 12, 864-866 (Nov 1987).

[16] Cobham Gaisler AB, GRLIB IP Core Users Manual, 2017.3 ed. (Nov 2017).

[17] RTEMS Project, RTEMS User Manual, 4.11.2 ed. (Jul 2017). 